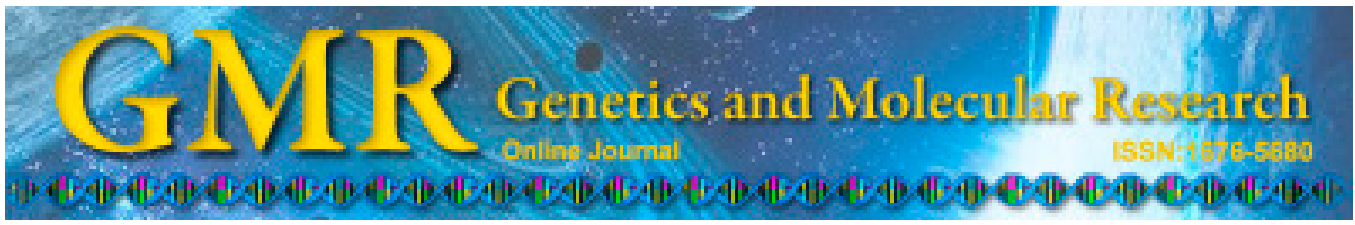

\title{
Association between TNFSF4 tagSNPs and myocardial infarction in a Chinese Han population
}

J. Cheng ${ }^{1 *}$, J.-M. Cen ${ }^{2 *}$, M.-Y. Cai ${ }^{1}$, S. Xu' ${ }^{1}$, L. Li ${ }^{1}$, Z.-C. Li ${ }^{1}$, X.-L. Yang ${ }^{2}$ C. Chen ${ }^{3}$, X. Liu ${ }^{1}$ and X.-D. Xiong ${ }^{1}$

${ }^{1}$ Institute of Aging Research, Guangdong Medical College, Dongguan, Guangdong, China

${ }^{2}$ Department of Cardiovascular Disease, The First People's Hospital of Foshan, Foshan, Guangdong, China

${ }^{3}$ Department of Cardiovascular Disease,

The Affiliated Hospital of Guangdong Medical College, Zhanjiang,

Guangdong, China

*These authors contributed equally to this study.

Corresponding author: X.-D. Xiong

E-mail: xiongxingdong@126.com

Genet. Mol. Res. 14 (2): 6136-6145 (2015)

Received July 21, 2014

Accepted December 3, 2014

Published June 8, 2015

DOI http://dx.doi.org/10.4238/2015.June.8.11

ABSTRACT. Tumor necrosis factor superfamily member 4 (TNFSF4) plays an important role in atherosclerosis development. However, the biological significance of TNFSF4 variants on myocardial infarction (MI) pathogenesis remains poorly understood. We investigated the influence of 5 TNFSF4 tagging single nucleotide polymorphisms (rs3861950, rs17346501, rs7518045, rs1234313, and rs3850641) on individual susceptibility to MI in a Chinese population of $285 \mathrm{MI}$ patients and 645 controls. Genotyping was performed using the polymerase chain reaction-ligase detection reaction method. In multivariate logistic regression analysis, only the TNFSF4 tagging single nucleotide polymorphism rs7518045 exhibited a significant effect on MI risk; A allele 
(odds ratio $=0.68,95 \%$ confidence interval $=0.46-1.00, \mathrm{P}=0.048)$ and AA genotype (odds ratio $=0.64,95 \%$ confidence interval $=0.42-0.97, \mathrm{P}$ $=0.036$ ) were associated with a decreased risk of MI compared with the $\mathrm{G}$ allele and the combined AG/GG genotype, respectively. Moreover, the haplotype rs3861950C-rs17346501C-rs7518045A-rs1234313G containing the rs7518045 A allele also exhibited a significant association with a decreased risk for MI (odds ratio $=0.42,95 \%$ confidence interval $=0.21-0.84, \mathrm{P}=0.011)$. Our study showed that the A allele of the rs7518045 and haplotype rs3861950C-rs17346501C-rs7518045Ars1234313G in the TNFSF4 gene were associated with decreased MI risk in a Chinese Han population. Further studies using larger sample sizes and in diverse ethnic populations are needed to confirm the general validity of our findings.

Key words: Myocardial infarction risk; Single nucleotide polymorphism; Tumor necrosis factor superfamily member 4

\section{INTRODUCTION}

Myocardial infarction (MI) is the leading cause of death in humans worldwide and its incidence is rapidly increasing (Watkins and Farrall, 2006). Numerous risk factors, including smoking, alcohol intake, diabetes, hypertension, hypercholesterolemia, obesity, physical inactivity, and psychosocial situation, have been reported to contribute to the pathogenesis of MI (Anand et al., 2008; Zhang et al., 2008). However, aside from these modifiable risk factors, accumulating studies have demonstrated that host genetic background plays a critical role on MI development (Zdravkovic et al., 2002; Kangas-Kontio et al., 2010).

Tumor necrosis factor superfamily member 4 (TNFSF4), also known as $O X 40 L$, is located in human chromosome 1 and encodes a type II glycoprotein that is expressed in various cells, including professional antigen-presenting cells, $\mathrm{CD} 4^{+} \mathrm{T}$ cells, $\mathrm{CD} 8^{+} \mathrm{T}$ cells, macrophages, dendritic cells, and vascular endothelial cells (Stuber et al., 1995; Imura et al., 1996; Ohshima et al., 1997; Murata et al., 2000). TNFSF4 functions as a ligand for TNFRSF4, and thus plays a crucial role in regulating $\mathrm{T}$ lymphocyte proliferation and survival, natural killer $\mathrm{T}$ cells and natural killer cell function, and regulatory T cell differentiation and activation (Ito et al., 2006; Duan et al., 2008). Accumulating experimental evidence has confirmed that activated T cells and inflammatory responses play an essential role in atherosclerosis development (Hansson, 2001; Hansson et al., 2002; de Boer et al., 2003). The inflammatory process is also largely responsible for plaque rupture and thrombus formation, which clinically manifests as ischemia, stroke, or MI (van der Wal et al., 1994; Nakajima et al., 2002; Hansson, 2005). In addition, TNFSF4-deficient mice had significantly smaller atherosclerotic lesions and higher levels of plasma total cholesterol and high-density lipoprotein cholesterol (HDL-C) than controls, while mice over-expressing TNFSF4 exhibited significantly larger atherosclerotic lesions (Wang et al., 2005). Interruption of the TNFSF4/TNFRSF4 pathway attenuates atherogenesis in low-density lipoprotein (LDL) receptor-deficient mice (van Wanrooij et al., 2007). Thus, the TNFSF4-TNFRSF4 pathway may play a key role in MI pathogenesis by participating in $\mathrm{T}$ cell activation and the inflammatory response.

Single nucleotide polymorphisms (SNPs) are the most frequent variants in the human 
genome and may contribute to individual susceptibility to some diseases. Increasing evidence suggests that SNPs within candidate genes may influence MI risk (Fujimaki et al., 2010; Ghaderian et al., 2010; Kallel et al., 2010). Wang et al. (2005) found that the TNFSF4 rs3850641 $\mathrm{G}$ allele contributed to the risk of developing MI in a Swedish population. Furthermore, the T allele of a promoter polymorphism (rs45454293), consistent with the linked rs3850641G-allele, was found to be associated with an increased risk of MI in Swedish women (Ria et al., 2011). To date, no study of TNFSF4 SNPs with MI risk in the Chinese population has been reported. Thus, we conducted a case-control study to elucidate the association between TNFSF4 polymorphisms and MI risk in a Chinese population. Because many SNPs have shown with correlated genotypes or linkage disequilibrium (LD), only a subset of SNPs (known as tagging SNPs, or tagSNPs) must be genotyped for disease association studies. In this study, we focused on 5 tagSNPs (rs3861950, rs17346501, rs7518045, rs1234313, and rs3850641) in the TNFSF4 gene.

\section{MATERIAL AND METHODS}

\section{Study subjects}

A total of 930 unrelated Chinese Han subjects were included in this study. A total of 285 patients with MI were recruited from the First People's Hospital of Foshan (Foshan, China) and the Affiliated Hospital of Guangdong Medical College (Zhanjiang, China) from March 2011 to July 2013. MI was diagnosed based on clinical symptoms and typical electrocardiographic changes, as well as on increases in the serum cardiac markers such as creatinine kinase, aspartate aminotransferase, lactate dehydrogenase, and troponin T. The diagnosis was confirmed by identifying the responsible stenosis in any of the major coronary arteries or in the left main trunk by coronary angiography. A total of 645 control subjects were consecutively recruited from the participating hospitals for regular physical examinations during the same period when MI patients were recruited. The unaffected controls were judged to be free of MI by questionnaires, medical history, clinical examination, and electrocardiography. Individuals with congestive heart failure, peripheral vascular disease, rheumatic heart disease, pulmonary heart disease, chronic kidney disease, hepatic disease, or any malignancy were excluded from the study.

All study subjects were interviewed after written informed consent was obtained, and structured questionnaires were administered by interviewers at enrollment to collect information on demographic data and risk factors related to MI. The study was approved by the Medical Ethics Committee of the First People's Hospital of Foshan and the Affiliated Hospital of Guangdong Medical College.

\section{Analysis of biochemical parameters}

An approximately $2 \mathrm{~mL}$ venous blood sample was drawn from each subject into tubes containing ethylenediaminetetraacetic acid after an overnight fast. The blood sample was centrifuged at $2000 \mathrm{~g}$ for $15 \mathrm{~min}$ immediately after collection and stored at $-80^{\circ} \mathrm{C}$ until analysis. The levels of plasma total cholesterol, triglyceride, HDL-C, and LDL-C were measured enzymatically using a chemistry analyzer (Olympus, Tokyo, Japan). Glucose was analyzed using the glucose oxidase method with an Abbott V/P Analyzer (Abbott Laboratories, Abbott Park, IL, USA). 


\section{DNA extraction}

Genomic DNA was extracted from peripheral whole blood using the TIANamp blood DNA extraction kit (TianGen Biotech, Beijing, China) according to manufacturer instructions. All DNA samples were dissolved in water and stored at $-20^{\circ} \mathrm{C}$ until use.

\section{TagSNP selection and genotyping}

The Chinese Han population's SNP data of the TNFSF4 gene were downloaded from the HapMap database (http://www.hapmap.org). We then analyzed these data using the Haploview software version 4.2 (Barrett et al., 2005). A minor allele frequency (MAF) $>0.05$ and LD measure $\left(\mathrm{r}^{2}\right)>0.8$ were prerequisites for tagSNP selection. We obtained 5 tagSNPs, including rs3861950, rs17346501, rs7518045, rs1234313, and rs3850641. Among the 5 tagSNPs, rs3861950, rs1234313, and rs3850641 had been previously investigated in 2 case-control samples from Sweden (Wang et al., 2005). The other 2 tagSNPs (rs17346501 and rs7518045) captured a broader extension (Table 1). Together, the 5 tagSNPs represented the information of more than $80 \%$ of TNFSF4 SNPs with an MAF $>0.05$ (Table 1). Haplotype analysis was performed using the SHEsis platform (Shi and $\mathrm{He}, 2005)$.

Genomic DNA was genotyped by polymerase chain reaction (PCR)-ligase detection reaction (LDR) method (Shanghai Biowing Applied Biotechnology Company, Shanghai, China). Primer and probe sequences are summarized in Table S1. PCR was carried out on the ABI 9600 (Applied Biosystems, Foster City, CA, USA) in a total volume of $20 \mu \mathrm{L}$, including $50 \mathrm{ng}$ genomic DNA, $1 \mathrm{X}$ PCR buffer, $3 \mathrm{mM} \mathrm{MgCl}, 2 \mathrm{mM}$ of each dNTP, 0.5 $\mathrm{mM} / \mathrm{mL}$ primer mix, and $1 \mathrm{U}$ hot-start Taq DNA polymerase (Qiagen, Hilden, Germany). Cycling parameters were as follows: $95^{\circ} \mathrm{C}$ for $2 \mathrm{~min} ; 40$ cycles at $94^{\circ} \mathrm{C}$ for $30 \mathrm{~s}, 56^{\circ} \mathrm{C}$ for $1 \mathrm{~min}$ and $30 \mathrm{~s}, 65^{\circ} \mathrm{C}$ for $30 \mathrm{~s}$; and a final extension step at $65^{\circ} \mathrm{C}$ for $10 \mathrm{~min}$. The ligation reaction for each PCR product was carried out in a final volume of $10 \mu \mathrm{L}$ containing $1 \mu \mathrm{L}$ $1 \mathrm{X}$ ligation buffer, $4 \mu \mathrm{L}$ PCR product, 2 pmol of each discriminating probe, 2 U Taq DNA ligase (New England Biolabs, Ipswich, MA, USA). The LDR parameters were as follows: $95^{\circ} \mathrm{C}$ for $2 \mathrm{~min}, 40$ cycles at $94^{\circ} \mathrm{C}$ for $15 \mathrm{~s}$, and $50^{\circ} \mathrm{C}$ for $25 \mathrm{~s}$. Following the LDR, $1 \mu \mathrm{L}$ LDR product was mixed with $1 \mu \mathrm{L}$ ROX and $1 \mu \mathrm{L}$ loading buffer. The mixture was then analyzed using an ABI Prism 377 DNA Sequencer (Applied Biosystems). Approximately $10 \%$ of the samples were randomly selected to perform repeated assays and the results were $100 \%$ concordant.

Table 1. Alleles captured by the 5 TNFSF4 tagSNPs.
\begin{tabular}{ll}
\hline tagSNPs & Alleles captured \\
\hline rs3861950 & rs7525284, rs7514229, rs4081545, rs10912564, rs 3861950 \\
rs17346501 & rs10912561, rs13343108, rs10489268, rs11808060, rs10798264, rs 17300347, rs 17346501, rs 17372309, rs 10912560 \\
rs7518045 & rs6661173, rs16845543, rs7518045, rs16845585, rs10489267 \\
rs1234313 & rs1234313 \\
rs3850641 & rs3850641 \\
\hline
\end{tabular}




\section{Statistical analysis}

The statistical power analysis was performed using Power and Sample size calculations, version 3.0.43 (Dupont and Plummer Jr., 1990). Both TNFSF4 tagSNPs were tested for conformity to Hardy-Weinberg equilibrium using a goodness-of-fit $\chi^{2}$ test among the control subjects. Quantitative variables are reported as means \pm standard deviation, and qualitative variables are reported as percentages. The differences in the demographic characteristics between the cases and controls were estimated using the $\chi^{2}$ test (for categorical variables) and the Student $t$-test (for continuous variables). For individual tagSNP association analyses, genotype frequencies were assessed using multivariate methods based on logistic regression analysis. We estimated odds ratios (ORs) and 95\% confidence intervals (CIs) for the effect of TNFSF 4 tagSNPs on MI risk adjusted by age, gender, smoking, drinking, hypertension, diabetes, and hyperlipidemia. Statistical analyses were performed using the SPSS software version 21 (SPSS, Inc., Chicago, IL, USA). Haplotype analysis on the polymorphisms was conducted using the SHEsis software (http://analysis.bio-x.cn/myAnalysis.php) (Shi and He, 2005). A P value of less than 0.05 was considered to be statistically significant.

\section{RESULTS}

\section{Characteristics of the study population}

Characteristics of patients with MI $(\mathrm{N}=285)$ and controls $(\mathrm{N}=645)$ are summarized in Table 2. There was no statistically significant difference between cases and controls in terms of age. Compared with the control group, more patients in the MI group were males, smokers, alcohol consumers, and individuals with hypertension, diabetes, or hyperlipidemia. The patient group also had significantly higher serum triglycerides, total cholesterol, and LDL-C, whereas serum HDL-C levels were significantly higher among controls. The average levels of systolic blood pressure, diastolic blood pressure, and fasting plasma glucose in the patients were significantly higher than those in controls. These data demonstrated that male gender, smoking, alcohol intake, hypertension, hyperlipidemia, and diabetes mellitus were important risk factors in the development of MI in our Chinese population.

Table 2. General characteristics in the MI cases and controls.

\begin{tabular}{lcrr}
\hline Variable & Controls $(\mathrm{N}=645)$ & MI $(\mathrm{N}=285)$ & P value $^{\mathrm{a}}$ \\
\hline Age (years) & $61.89 \pm 11.95$ & $62.07 \pm 11.99$ & 0.493 \\
Gender (male) & $380(58.0 \%)$ & $221(77.5 \%)$ & $<0.001$ \\
Smoking & $169(25.8 \%)$ & $171(60.0 \%)$ & $<0.001$ \\
Drinking & $95(14.5 \%)$ & $77(27.0 \%)$ & $<0.001$ \\
Hypertension & $233(35.6 \%)$ & $179(62.8 \%)$ & $<0.001$ \\
Diabetes & $106(16.2 \%)$ & $136(47.7 \%)$ & $<0.001$ \\
Hyperlipidemia & $247(37.7 \%)$ & $201(70.5 \%)$ & $<0.001$ \\
Systolic blood pressure (mmHg) & $132.55 \pm 18.94$ & $140.02 \pm 19.16$ & $<0.001$ \\
Diastolic blood pressure (mmHg) & $72.84 \pm 10.46$ & $75.66 \pm 11.56$ & $<.501$ \\
FPG (mM) & $5.82 \pm 1.92$ & $6.64 \pm 1.72$ & $<0.001$ \\
TG (mM) & $1.49 \pm 0.82$ & $2.06 \pm 0.97$ & $<.001$ \\
TC (mM) & $4.63 \pm 1.16$ & $4.71 \pm 1.21$ & \\
HDL-C (mM) & $1.37 \pm 0.67$ & $1.18 \pm 0.36$ & $<0.297$ \\
LDL-C (mM) & $2.64 \pm 0.92$ & $3.03 \pm 0.97$ & $<0.001$ \\
\hline
\end{tabular}

${ }^{a}$ Two-sided chi-square test or independent-sample Student $t$-test. FPG, fasting blood glucose; TG, triglycerides; TC, total cholesterol; HDL-C, high-density lipoprotein cholesterol; LDL-C, low-density lipoprotein cholesterol. 


\section{Multivariate association between TNFSF4 tagSNPs and MI risk}

Five TNFSF4 tagSNPs (rs3861950, rs17346501, rs7518045, rs1234313, and rs3850641) were genotyped in 285 MI patients and 645 control subjects. The primary information for the tagSNPs is shown in Table 3. The MAF of these tagSNPs in our controls was similar to the MAF in the Chinese in HapMap database (Table 3). The genotype frequency distributions of the tagSNPs in our control subjects were in Hardy-Weinberg equilibrium (all $P$ values $\geq 0.05$; Table 3 ).

Table 3. Primary information for the 5 TNFSF4 tagSNPs.
\begin{tabular}{lccccc} 
Genotyped & $\begin{array}{c}\text { Chr Pos } \\
\text { tagSNPs }\end{array}$ & $\begin{array}{c}\text { Pos in } \\
\text { (Genome Build 106) }\end{array}$ & $\begin{array}{c}\text { MAF for Chinese } \\
\text { TNFSF gene }\end{array}$ & $\begin{array}{c}\text { MAF in our } \\
\text { (CHB) in HapMap }\end{array}$ & $\begin{array}{c}\text { P value for HWE } \\
\text { test in our controls }\end{array}$ \\
\hline rs3861950 & 173187153 & Intron 2 & 0.128 & 0.138 & 0.058 \\
rs17346501 & 173188402 & Intron 2 & 0.057 & 0.040 & 0.333 \\
rs7518045 & 173194373 & Intron 1 & 0.067 & 0.091 & 0.198 \\
rs1234313 & 173197108 & Intron 1 & 0.326 & 0.376 & 0.328 \\
rs3850641 & 173206693 & Intron 1 & 0.107 & 0.215 & 0.769 \\
\hline
\end{tabular}

${ }^{a} \mathrm{MAF}$ : minor allele frequency. ${ }^{\mathrm{b}} \mathrm{HWE}$ : Hardy-Weinberg equilibrium.

Associations between the 5 tagSNPs and MI were analyzed using multivariate logistic regression analysis after adjusting for age, gender, smoking, drinking, hypertension, diabetes, and hyperlipidemia as covariates. The allele and genotype distributions of the tagSNPs in the cases and controls are listed in Table 4. Only rs7518045 showed significant differences in allele frequency and genotype distribution between MI patients and control subjects. The frequency of the rs $7518045 \mathrm{~A}$ allele in cases was significantly lower than that in control subjects, indicating that the A allele may be associated with a lower risk of $\mathrm{MI}(\mathrm{OR}=0.68,95 \% \mathrm{CI}=0.46-1.00, \mathrm{P}=0.048$; Table 4$)$. Analysis using multivariate logistic regression revealed a similar trend for the decreased risk of $\mathrm{MI}$ in the dominant model in which the $\mathrm{AG}$ and $\mathrm{GG}$ genotypes were combined $(\mathrm{OR}=0.64,95 \% \mathrm{CI}$ $=0.42-0.97, \mathrm{P}=0.036$; Table 4 ). We further evaluated the genotypes and MI susceptibility after stratifying the subjects by gender, age, and smoking or drinking status. However, no additional evident association between rs7518045 and MI risk was observed among subgroups by age, smoking status, etc. (data not shown).

\section{Association between haplotypes of TNFSF4 tagSNPs and MI risk}

LD analysis for the 5 tagSNPs was performed using the Haploview platform, and showed that 4 tagSNPs (rs3861950, rs17346501, rs7518045, and rs1234313) were located in 1 haplotypic block and could be combined to construct haplotypes (Figure 1). Thus, we further compared the haplotype frequency between MI and controls. Four common haplotypes (frequency $>3 \%$ ) derived from the 4 tagSNPs accounted for nearly $100 \%$ of the haplotype variations. Among the 4 common haplotypes, only the haplotype rs3861950C-rs17346501Crs $7518045 \mathrm{~A}-\mathrm{rs} 1234313 \mathrm{G}$ was found to be associated with a decreased risk of $\mathrm{MI}(\mathrm{OR}=0.42$, $95 \% \mathrm{CI}=0.21-0.84, \mathrm{P}=0.011$; Table 5). 
Table 4. Genotypic and allelic frequencies of TNFSF4 tagSNPs and MI risk.

\begin{tabular}{|c|c|c|c|c|c|}
\hline \multirow[t]{2}{*}{ Genotyped tagSNPs } & \multirow[t]{2}{*}{ Genotype or allele } & Controls $(\mathrm{N}=645)$ & Cases $(\mathrm{N}=285)$ & \multirow[t]{2}{*}{ OR $(95 \% \mathrm{CI})$} & \multirow[t]{2}{*}{$\overline{\text { P value }}{ }^{a}$} \\
\hline & & $\mathrm{N}(\%)$ & $\mathrm{N}(\%)$ & & \\
\hline \multirow[t]{4}{*}{ rs3861950 } & $\mathrm{T}$ & $1112(86.2)$ & $495(86.8)$ & 1.00 & - \\
\hline & $\mathrm{C}$ & $178(13.8)$ & $75(13.2)$ & $0.89(0.64-1.23)$ & 0.470 \\
\hline & TT & 485 (75.2) & $214(75.1)$ & 1.00 & - \\
\hline & $\mathrm{CT}+\mathrm{CC}$ & $160(24.8)$ & $71(24.9)$ & $1.25(0.85-1.83)$ & 0.255 \\
\hline \multirow[t]{4}{*}{ rs17346501 } & $\mathrm{T}$ & $1238(96.0)$ & $560(98.2)$ & 1.00 & - \\
\hline & $\mathrm{C}$ & $52(4.0)$ & $10(1.8)$ & $0.52(0.24-1.12)$ & 0.093 \\
\hline & TT & $595(92.2)$ & $275(96.5)$ & 1.00 & - \\
\hline & $\mathrm{CT}+\mathrm{CC}$ & $50(7.8)$ & $10(3.5)$ & $0.52(0.24-1.14)$ & 0.101 \\
\hline \multirow[t]{4}{*}{ rs7518045 } & G & $117(9.1)$ & $61(10.7)$ & 1.00 & - \\
\hline & A & $1173(90.9)$ & $509(89.3)$ & $0.68(0.46-1.00)$ & 0.048 \\
\hline & $\mathrm{AG}+\mathrm{GG}$ & $109(16.9)$ & $58(20.4)$ & 1.00 & - \\
\hline & $\mathrm{AA}$ & $536(83.1)$ & 227 (79.6) & $0.64(0.42-0.97)$ & 0.036 \\
\hline \multirow[t]{4}{*}{ rs1234313 } & A & $805(62.4)$ & $356(62.5)$ & 1.00 & - \\
\hline & G & 485 (37.6) & $214(37.5)$ & $0.98(0.77-1.25)$ & 0.878 \\
\hline & $\mathrm{AA}$ & $257(39.8)$ & $112(39.3)$ & 1.00 & - \\
\hline & $\mathrm{AG}+\mathrm{GG}$ & $388(60.2)$ & $173(60.7)$ & $1.02(0.73-1.43)$ & 0.914 \\
\hline \multirow{4}{*}{ rs3850641 } & $\mathrm{G}$ & $277(21.5)$ & $126(22.1)$ & 1.00 & - \\
\hline & A & $1013(78.5)$ & 444 (77.9) & $0.82(0.63-1.08)$ & 0.164 \\
\hline & GG & $31(4.8)$ & $19(6.7)$ & 1.00 & - \\
\hline & $\mathrm{AG}+\mathrm{AA}$ & $614(95.2)$ & $266(93.3)$ & $0.55(0.28-1.08)$ & 0.083 \\
\hline
\end{tabular}

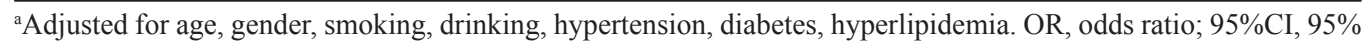
confidence interval.

A

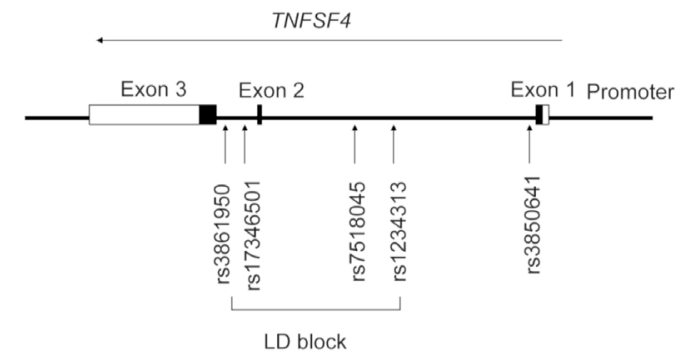

B

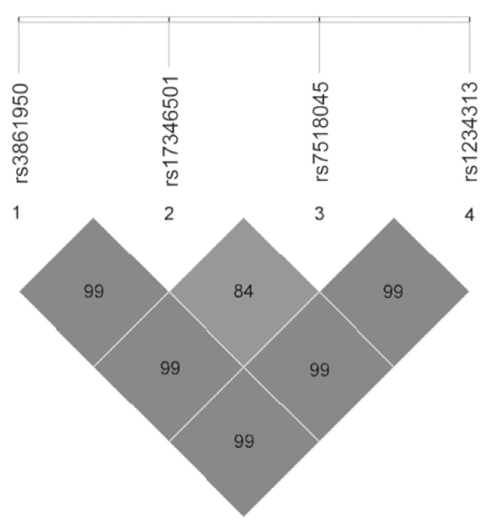

Figure 1. A. Positions of the TNFSF4 tagSNPs genotyped in the present study. B. Linkage disequilibrium analysis among the 4 TNFSF4 tagSNPs located in one haplotypic block. Numbers within squares indicate the D' value reported as a percentile. 
Table 5. Association between haplotypes of rs3861950, rs17346501, rs7518045, and rs1234313 and MI risk.

\begin{tabular}{lcccr}
\hline Haplotype & Controls $(\mathrm{N}=645)$ & Cases $(\mathrm{N}=285)$ & \multirow{2}{*}{ OR (95\%CI) } & P value \\
\cline { 2 - 3 } & $\mathrm{N}(\%)$ & $\mathrm{N}(\%)$ & & \\
\hline rs3861950C-rs17346501C-rs7518045A-rs1234313G & $52.00(4.0)$ & $9.99(1.8)$ & $0.42(0.21-0.84)$ & 0.011 \\
rs3861950C-rs17346501T-rs7518045G-rs1234313G & $115.99(9.0)$ & $61.00(10.7)$ & $1.21(0.87-1.68)$ & 0.251 \\
rs3861950T-rs17346501T-rs7518045A-rs1234313A & $804.99(62.4)$ & $355.99(62.5)$ & $1.00(0.81-1.23)$ & 0.986 \\
rs3861950T-rs17346501T-rs7518045A-rs1234313G & $306.00(23.7)$ & $139.01(24.4)$ & $1.04(0.82-1.30)$ & 0.769 \\
\hline
\end{tabular}

$\mathrm{OR}=$ odds ratio; $95 \% \mathrm{CI}, 95 \%$ confidence interval.

\section{DISCUSSION}

The principal pathogenesis of MI is coronary atherosclerotic plaque formation and rupture. Recent studies have provided evidence that the TNFSF4-TNFRSF4 pathway plays a key role in MI pathogenesis by participating in T cell activation and the inflammatory response. However, the association between polymorphisms in the TNFSF4 gene and MI risk remains unknown. In the present study, we performed a genetic association analysis on 5 TNFSF4 tagSNPs (rs3861950, rs17346501, rs7518045, rs1234313, and rs3850641) in $285 \mathrm{MI}$ patients and 645 controls. We found that only rs 7518045 had a significant effect on MI risk. In addition, the haplotype rs3861950C-rs17346501C-rs7518045A-rs1234313G containing the rs7518045 A allele also exhibited a significant association with a decreased risk of MI.

Despite the association between rs7518045 and decreased MI risk, the underlying mechanism of the rs 7518045 polymorphism influencing disease susceptibility remains unclear. Because the rs 7518045 SNP is located in intron 1, it is not expected to change the functional properties of the TNFSF4 protein. However, rs7518045 is a tagSNP, and the haplotype block in which it lies also covers exon 2 of the gene. Thus, this SNP is in LD with a causal SNP that may either result in a change in amino acid or affect a possible splice variant or the stability of the mRNA. Future fine mapping and re-sequencing of the TNFSF4 gene may detect such functional variants. Further studies involving large samples and more variations are warranted to clarify the impact of the gene on MI risk.

With respect to the tagSNPs rs3861950, rs17346501, rs1234313, and rs3850641 of the TNFSF4 gene, we observed no association between MI risk in the Chinese Han population. This result agrees with two previous reports that showed no evidence of an association between TNFSF4 gene variation (5 SNPs, including rs3861950 and rs1234313) and the risk for coronary heart disease or MI (Koch et al., 2008; Cheng et al., 2011). However, the minor allele of rs3850641 was more significantly frequent in individuals with MI than in the controls in 2 independent populations from Sweden (Wang et al., 2005), while no significant difference was observed in a study by Cheng et al. (2011) and our studies in the Chinese Han population, as well as in a study by Koch et al. (2008) in a German population. The main reason for this discrepancy may be because of varying genetic backgrounds, differences between the studies in geographical ethnic groups, sample size, and study design.

There were several limitations to our study. First, the patients and controls were enrolled from hospitals and may not represent the general population. Nonetheless, the genotype distribution of the controls was in Hardy-Weinberg equilibrium. Second, the moderate sample size limited the statistical power of our study, particularly in the case subjects. Third, based on functional considerations, the tagSNPs investigated were non-coding, and we therefore assume these variants to be linked with 1 or more functional variants within the TNFSF4 gene 
or its regulatory regions. Finally, further studies in different population may establish the true significance of the association between these SNPs and MI risk.

In summary, the tagSNP rs7518045 and the haplotype rs3861950C-rs17346501Crs $7518045 \mathrm{~A}-\mathrm{rs} 1234313 \mathrm{G}$ were associated with a decreased risk of MI, suggesting that these genetic variants can be used as biomarkers for assessing the risk of developing MI. Further studies with larger sample sizes and in diverse ethnic populations are necessary to confirm the general validity of our findings.

\section{Conflicts of interest}

The authors declare no conflict of interest.

\section{ACKNOWLEDGMENTS}

Research supported by grants from the National Natural Science Foundation of China (\#81370456), the Natural Science Foundation of Guangdong Province (\#S2012010008219), and the Science and Technology Planning Project for University Research Institutions and Medical and Health Organizations of Dongguan City (\#2011105102007).

\section{Supplementary material}

\section{REFERENCES}

Anand SS, Islam S, Rosengren A, Franzosi MG, et al. (2008). Risk factors for myocardial infarction in women and men: insights from the INTERHEART study. Eur. Heart J. 29: 932-940.

Barrett JC, Fry B, Maller J and Daly MJ (2005). Haploview: analysis and visualization of LD and haplotype maps. Bioinformatics 21: 263-265.

Cheng G, Wang H, Chen M, Li L, et al. (2011). Lack of evidence to support the association of polymorphisms within the TNFSF4 gene and coronary heart disease in a Chinese Han population. Exp. Ther. Med. 2: 275-280.

de Boer OJ, Becker AE and van der Wal AC (2003). T lymphocytes in atherogenesis-functional aspects and antigenic repertoire. Cardiovasc. Res. 60: 78-86.

Duan W, So T and Croft M (2008). Antagonism of airway tolerance by endotoxin/lipopolysaccharide through promoting OX40L and suppressing antigen-specific Foxp3+ T regulatory cells. J. Immunol. 181: 8650-8659.

Dupont WD and Plummer WD Jr (1990). Power and sample size calculations. A review and computer program. Control Clin. Trials 11: 116-128.

Fujimaki T, Kato K, Yokoi K, Oguri M, et al. (2010). Association of genetic variants in SEMA3F, CLEC16A, LAMA3, and PCSK2 with myocardial infarction in Japanese individuals. Atherosclerosis 210: 468-473.

Ghaderian SM, Akbarzadeh Najar R and Tabatabaei Panah AS (2010). Genetic polymorphisms and plasma levels of matrix metalloproteinases and their relationships with developing acute myocardial infarction. Coron. Artery Dis. 21: $330-335$

Hansson GK (2001). Immune mechanisms in atherosclerosis. Arterioscler. Thromb. Vasc. Biol. 21: 1876-1890.

Hansson GK (2005). Inflammation, atherosclerosis, and coronary artery disease. N. Engl. J. Med. 352: 1685-1695.

Hansson GK, Libby P, Schonbeck U and Yan ZQ (2002). Innate and adaptive immunity in the pathogenesis of atherosclerosis. Circ. Res. 91: 281-291.

Imura A, Hori T, Imada K, Ishikawa T, et al. (1996). The human OX40/gp34 system directly mediates adhesion of activated T cells to vascular endothelial cells. J. Exp. Med. 183: 2185-2195.

Ito T, Wang YH, Duramad O, Hanabuchi S, et al. (2006). OX40 ligand shuts down IL-10-producing regulatory T cells. Proc. Natl. Acad Sci. U S A 103: 13138-13143.

Kallel A, Sediri Y, Sbai MH, Mourali MS, et al. (2010). The paraoxonase L55M and Q192R gene polymorphisms and myocardial infarction in a Tunisian population. Clin. Biochem. 43: 1461-1463.

Kangas-Kontio T, Huotari A, Ruotsalainen H, Herzig KH, et al. (2010). Genetic and environmental determinants of total 
and high-molecular weight adiponectin in families with low HDL-cholesterol and early onset coronary heart disease. Atherosclerosis 210: 479-485.

Koch W, Hoppmann P, Mueller JC, Schomig A, et al. (2008). Lack of support for association between common variation in TNFSF4 and myocardial infarction in a German population. Nat. Genet. 40: 1386-1387; author reply 1387-1388.

Murata K, Ishii N, Takano H, Miura S, et al. (2000). Impairment of antigen-presenting cell function in mice lacking expression of OX40 ligand. J. Exp. Med. 191: 365-374.

Nakajima T, Schulte S, Warrington KJ, Kopecky SL, et al. (2002). T-cell-mediated lysis of endothelial cells in acute coronary syndromes. Circulation 105: 570-575.

Ohshima Y, Tanaka Y, Tozawa H, Takahashi Y, et al. (1997). Expression and function of OX40 ligand on human dendritic cells. J. Immunol. 159: 3838-3848.

Ria M, Lagercrantz J, Samnegard A, Boquist S, et al. (2011). A common polymorphism in the promoter region of the TNFSF4 gene is associated with lower allele-specific expression and risk of myocardial infarction. PLoS One 6: e17652.

Shi YY and He L (2005). SHEsis, a powerful software platform for analyses of linkage disequilibrium, haplotype construction, and genetic association at polymorphism loci. Cell Res. 15: 97-98.

Stuber E, Neurath M, Calderhead D, Fell HP, et al. (1995). Cross-linking of OX40 ligand, a member of the TNF/NGF cytokine family, induces proliferation and differentiation in murine splenic B cells. Immunity 2: 507-521.

van der Wal AC, Becker AE, van der Loos CM and Das PK (1994). Site of intimal rupture or erosion of thrombosed coronary atherosclerotic plaques is characterized by an inflammatory process irrespective of the dominant plaque morphology. Circulation 89: 36-44.

van Wanrooij EJ, van Puijvelde GH, de Vos P, Yagita H, et al. (2007). Interruption of the Tnfrsf4/Tnfsf4 (OX40/OX40L) pathway attenuates atherogenesis in low-density lipoprotein receptor-deficient mice. Arterioscler. Thromb. Vasc. Biol. 27: 204-210.

Wang X, Ria M, Kelmenson PM, Eriksson P, et al. (2005). Positional identification of TNFSF4, encoding OX40 ligand, as a gene that influences atherosclerosis susceptibility. Nat. Genet. 37: 365-372.

Watkins H and Farrall M (2006). Genetic susceptibility to coronary artery disease: from promise to progress. Nat. Rev. Genet. 7: 163-173.

Zdravkovic S, Wienke A, Pedersen NL, Marenberg ME, et al. (2002). Heritability of death from coronary heart disease: a 36-year follow-up of 20,966 Swedish twins. J. Intern. Med. 252: 247-254.

Zhang XH, Lu ZL and Liu L (2008). Coronary heart disease in China. Heart 94: 1126-1131. 